Smith, Stanley

\section{Gene expression profiling in a model of human neuronal differentiation using oligonucleotide arrays}

\author{
Stanley C. Smith ${ }^{1}$, Stefan Pzyborski ${ }^{1}$, Christopher Aston ${ }^{1}$, Aiqing \\ $\mathrm{He}^{1}$, Anne Marie Velasco', Greg Tucker Kellogg' ${ }^{2}$, Peter Andrew ${ }^{3}$ \\ \& Andrew Wood ${ }^{1}$ \\ ${ }^{1}$ Neuroscience Research, Wyeth-Ayerst Research, \\ Princeton, New Jersey 08543, USA \\ ${ }^{2}$ Genetics Institute, Cambridge, M assachusetts 02140, USA \\ ${ }^{3}$ University of Sheffield, Department of Biomedical Science, Western Bank \\ Sheffield, S10 2TN, UK
}

In an effort to understand the process of human neuronal differentiation, we have monitored gene expression in a cell culture model, NTERA2/D1 (NT2). This pluripotent human teratinocarcinoma cell line was induced to differentiate with retinoic acid (RA) over a 21-day period. We monitored gene expression at four time points using a custom-fabricated oligonucleotide GenechipÒ. A panel of human genes that were expected to participate in the process of neurogenesis or act as differentiation markers were chosen and FASTA analysis determined regions of unique sequence. Oligonucleotide selection, masking and fabrication of GenechipsÒ were performed at Affymetrix. Arrays contained 50 probe pairs per gene and 589 genes per chip, as well as controls (probe pairs for housekeeping genes and spiked samples). We analysed data using GenechipÒ software and performed pairwise comparison between untreated NT2 at 0 days and RA-treated NT2 at 3, 7, 14 and 21 days. Increased and decreased expression of select genes was seen throughout the time course. The largest differences observed were in the order of a 50-fold difference from control. As expected, expression of the intermediate filament neuroepithelial marker nestin increased rapidly following RAtreatment and decreased toward the end of the time course. This regulation likely reflects the appearance and differentiation of neuronal precursors in the cultures. These data suggest that the experimental design and analysis techniques were valid. Expression of Hox genes A1, A4, B2, B3, B5 and C5 were greatly altered throughout the time course, as were expression of helix-loop-helix domain-containing genes important to the process of neuronal specification. The ability to study the coordinate regulation of such genes with oligonucleotide arrays may enable signal transduction pathways associated with human neurogenesis and differentiation to be deciphered.

Soares, Marcelo

\section{A rat heart UniGene set for identification of spatially and/or temporally regulated genes during heart development}

\author{
Jennifer Laffin, Shereen Chang, Todd Scheetz, Kyle M unn, Chad \\ Roberts, Kang Liu, Jim Lin, Thomas Casavant \& M arcelo Bento \\ Soares
}

Departments of Pediatrics, Physiology and Biophysics, Electrical and Computer Engineering, The University of lowa, lowa City, lowa, USA

Mammalian cardiac morphogenesis involves a number of fundamental processes, which are as yet poorly understood at the molecular level, such as the commitment of mesodermic cells to cardiogenic cells, the fusion of cardiogenic mesoderm into a tubular heart, the rightward looping of the tubular heart, the formation of valves and septa, the chamber specification, the trabeculation of myocardium, the vasculogenesis, etc. The resulting cardiovascular system comprises a diversity of cell types, which need to work in concert to generate normal cardiovascular function.
Among those are muscle cells critical for producing and maintaining pulsatile flow, neuroendocrine cells involved in control and regulation, endothelial cells lining the vascular tree and matrix secretory cells responsible for the deposition and upkeep of fibrous and elastic components of the system. Genes with a spatial and/or temporal pattern of expression during heart development are anticipated to play an important role in the formation of a functioning heart. Thus, the primary objective of our project is to generate a comprehensive catalogue of genes expressed during development of the rat heart, and to conduct microarray hybridisation experiments to identify a subset of spatially and/or temporally regulated genes. Our ultimate goal is to contribute resources that might facilitate research aimed at understanding the molecular mechanisms underlying heart development and congenital heart diseases. To date we have generated a rat heart UniGene set comprising 5,000 unique 3'ESTs from a total of 11,900 3'ESTs derived from a number of non-normalised and normalised adult and embryonic heart cDNA libraries. More specifically, ESTs were generated from cDNA libraries made from adult heart, $13 \mathrm{dpc}$ ventricles, $15 \mathrm{dpc}$ atria, $15 \mathrm{dpc}$ ventricles, $15 \mathrm{dpc}$ AV canal, 17 $\mathrm{dpc}$ atria, $17 \mathrm{dpc}$ ventricles and $17 \mathrm{dpc} \mathrm{AV}$ canal. Microarray hybridisation will be performed with cDNA targets derived from RNA obtained from each region of the heart throughout development for identification of spatially and/or temporally regulated genes.

Somme, Stig

\section{Differential gene expression in colorectal cancer of either the ascending or the descending colon}

\author{
Stig Somme \& Karl-Erik Giercksky
}

The Norwegian Radium Hospital, The University of O slo School of M edicine, Norway

Colorectal cancer is one of the most common cancers affecting both women and men; it kills approximately $50 \%$ of patients and this number has been almost unchanged for 20 years. Gene expression arrays are promising instruments for the study of gene expression in cancer. This high-throughput technology offers an advantage to more conventional techniques because of the large number of genes that are studied simultaneously. This offers insight into up- and downregulation of multiple genes. Gene expression profiling can help to characterize colorectal cancer and provide information on therapeutic gene targeting if this becomes available as a treatment modality. We have developed a method to study the differential expression of genes in colorectal cancer of either the ascending or descending colon by using mouse xenografts and gene expression arrays. We developed xenografts from patients with colorectal cancer of either the ascending or descending colon. Gene expression arrays were used to study the differential expression of 588 genes in two ascending colon cancer xenografts and two descending colon cancer xenografts. These results were compared with the expression profile of normal colon mucosa. We identified a significant upregulation ( $>3$ times) of 3-9 genes in the 4 xenografts. We also identified a significant downregulation ( $>3$ times) of 48-74 genes in the xenografts compared with the normal mucosa. To supplement the results, we have constructed a database, accessible via the Internet. This study demonstrates how expression arrays in combination with animal models can be used to study differential gene expression in colorectal cancer. The large amount of data obtained from this study offers both a challenge and an opportunity to better understand why colon cancer of the ascending colon prognostically and epidemiologically differs from that of the descending colon. 